\title{
Anxiety sensitivity and pain: generalisability across noxious stimuli
}

\author{
Trevor Thompson ${ }^{\mathrm{a}}$, Edmund Keogh ${ }^{\mathrm{b}}$, Christopher C. French $^{\mathrm{a}}$, Robert Davis ${ }^{\mathrm{a}}$ \\ ${ }^{\mathrm{a}}$ Department of Psychology, Goldsmiths College, University of London, New Cross, London SE14 6NW, UK \\ ${ }^{b}$ Department of Psychology, University of Bath, Claverton Down, Bath BA2 7AY, UK
}

Corresponding author: Trevor Thompson. Tel: +44-207-919-7171; fax: +44-207-919-7873.

Email address: t.thompson@gold.ac.uk

Keywords: anxiety sensitivity; pain; sex differences; cold pressor; thermal heat

Number of text pages (including figures and tables): 34

Number of Tables: 2

Number of Figures: 3 


\begin{abstract}
Anxiety sensitivity, a fear of anxiety-related symptoms, has been associated with a heightened experience of pain, especially within women. The majority of experimental studies investigating this association have relied heavily on the cold pressor technique as a means of pain induction, limiting the generalisability of results. The aim of the current study was to extend previous research by using two types of pain stimuli (cold and heat) to determine whether the link between anxiety sensitivity and pain generalises beyond cold pressor pain. The pain experience of 125 participants in response to these stimuli was assessed using threshold and tolerance readings, as well as subjective pain ratings. Results indicated a positive association between anxiety sensitivity and subjective pain, with this association observed primarily in females. Although analysis also indicated a basic generalisability of results across pain stimuli, anxiety sensitivity effects appeared to be especially pronounced during heat stimulation. These findings suggest that those high in anxiety sensitivity may respond more negatively to specific types of pain. Possible implications along with suggestions for future research are discussed.
\end{abstract}




\section{Introduction}

Anxiety sensitivity is defined as a trait tendency to be fearful of anxiety-related symptoms (Reiss et al., 1986), and has been associated with more negative pain experiences in both chronic (e.g., Asmundson and Norton, 1995; Plehn et al., 1998) and acute settings (Lang et al., 2005). Similar findings have also been reported in laboratory studies in healthy volunteers. Keogh and colleagues, for example, found that anxiety sensitivity was positively associated with sensory and affective pain ratings in response to the cold pressor task (Keogh and Mansoor, 2001; Keogh and Cochrane, 2002). Interestingly, a study by Keogh and Birkby (1999) found that anxiety sensitivity may have a sex-specific effect, with anxiety sensitivity differences in sensory ratings of cold pain found in females, but not in males.

In identifying a link between anxiety sensitivity and pain, the majority of these laboratorybased studies have tended to rely on a single method of noxious stimulation - the cold pressor task. However, as Janal et al. (1994) note, findings obtained using a specific pain modality have not always generalised to other pain modalities. Indeed, pain ratings compared across noxious stimuli such as cold pressor, heat, electrical and pressure pain responses have often demonstrated weak or even no correlation (Davidson and McDougall, 1969; Lynn and Perl, 1977; Janal et al., 1994). Furthermore, Riley et al. (1998) found that while sex differences in pain tolerance were large for pressure pain, they were often small to moderate for noxious heat stimuli. More recently, Jones et al. (2003) found a positive association between trait anxiety and pain intensity during cold stimulation but no such association during heat stimulation. Such a comparison across pain types has not yet been made within the context of anxiety sensitivity, which is empirically and conceptually distinct from trait anxiety (Taylor, 1999). 
The available data suggest that the investigation of anxiety sensitivity in relation to other types of pain induction is critical to determining the generalisability of effects. The primary aim of the present study, therefore, was to examine whether the relationship between anxiety sensitivity and pain was consistent across different types of pain stimuli. For the purposes of the current study, we chose to compare two related, yet different, types of pain (heat and cold) in healthy men and women who varied in levels of anxiety sensitivity. The specific predictions were that:

(1) Females would demonstrate greater pain sensitivity than males

(2) Anxiety sensitivity and pain would be positively related, with this relationship expected to be particularly pronounced in females

(3) The two relationships above would generalise across cold and heat modalities

\section{Method}

\section{Design}

A regression design was employed. The two predictor variables were anxiety sensitivity and sex. The dependent variables were five measures of pain experience: two behavioural pain measures (pain threshold and tolerance time), and three subjective pain rating scales from the SF-MPQ (Melzack, 1987). Order of pain stimuli was counterbalanced within gender, with half of the participants receiving the cold followed by the heat stimulus, with this order reversed for the remaining half. Resting blood pressure, menstrual phase and negative mood state were also recorded, as previous research has suggested that these may operate as potential process variables underlying sex and anxiety sensitivity differences in pain (Fillingim and Maixner, 1996; Keogh and Birkby, 1999; Riley et al., 1999). 


\section{$\underline{\text { Participants }}$}

The sample consisted of 125 adult participants ( 37 males and 88 females) with an overall average age of $23.9(\mathrm{sd}=6.5)$. Participants were primarily Goldsmiths College undergraduate students recruited via the University's course credits scheme, with the remainder paid volunteers (£5) who responded to advertisements posted around the university. The only exclusion criterion was the presence of any condition that might have interfered with the detection of pain sensations (e.g., Raynaud's disease, arthritis, cardiovascular disease, etc.).

\section{Pain Induction}

Two methods of pain induction were used in the current study: thermal cold and thermal heat. Thermal cold pain was induced using the cold-pressor task. This method was selected because of its high reliability and validity (Wolff, 1984; Edens and Gil, 1995) and to allow comparison with previous studies of anxiety sensitivity and cold pain (Keogh and Birkby, 1999; Keogh and Mansoor, 2001; Keogh and Cochrane, 2002). To provide a common baseline, participants first placed their non-dominant hand in a warm water tank $\left(37^{\circ} \mathrm{C}\right)$ for 2 minutes. Participants then transferred their hand to a cold-water tank maintained at a temperature of $1.5^{\circ} \mathrm{C}\left(+/-0.2^{\circ} \mathrm{C}\right)$, with a motor providing circulation of the water to prevent localised heating around the immersed hand. Participants reported when they first felt pain ('threshold'), and when they were no longer able to tolerate the pain ('tolerance') at which point they removed their hand. An upper time limit of two minutes was imposed as around $8 \%$ of men and $4 \%$ of women appear to adapt to the increasing numbing effect of the cold without reporting pain (Wolff, 1984). Participants were unaware of the time limit prior to the pain task.

For the thermal heat induction, a peltier-based thermode with a $5 \mathrm{~cm} \times 5 \mathrm{~cm}$ aluminum contact pad was used. After a visual demonstration by the experimenter, participants were required to 
undergo one practice trial and one experimental trial. For the practice trial, participants rested the thenar eminence of one hand (randomly selected) on the contact pad for 30s, with the pad maintained at a constant temperature of $40^{\circ} \mathrm{C}$. This practice trial served to eliminate any substantial differences in baseline hand temperature across participants, and to familiarise participants with the heat sensation and thus reduce the likelihood of pre-tolerance instinctive withdrawal on the subsequent experimental trial. For the experimental trial, the pad was maintained at a constant temperature of $48^{\circ} \mathrm{C}$, with participants reporting threshold and tolerance in accordance with the protocol of the cold pressor task. An upper time limit of 10 seconds was imposed, with this limit established as appropriate during pilot testing. Participants were not made aware of this time limit prior to the task.

\section{Questionnaires}

A battery of measures was administered, with those relevant to the current study reported below:

Demographics: This contained items relating to age and sex. For females, menstrual phase data based on the estimated number of days since the start of the last/current period was also collected. Responses of 0-14 days were classified as 'follicular' with 15+ days classified as 'luteal' (Riley et al., 1999). Oral contraceptive use (pill/no pill) was also recorded.

Short Form - McGill Pain Questionnaire (SF-MPQ; Melzack, 1987): The SF-MPQ is designed to provide a comprehensive assessment of the sensory and affective dimensions of pain as well as the overall evaluation of pain intensity. The sensory pain scale consists of 11 sensory descriptors (e.g. 'throbbing'), and the affective pain scale of 4 affective descriptors (e.g. 'sickening'). Each descriptor is rated on a discrete 4-point scale from none (0) to severe (3). 
Overall sensory and affective pain scores can be calculated by aggregating scores on each subscale. A Present Pain Index (PPI) is also included which attempts to assess the overall level of pain intensity on a six-point scale ranging from 0 ('no pain') to 5 ('excruciating pain'). The SF-MPQ correlates very highly with the full-length MPQ (Dudgeon et al., 1993) and is sensitive to change brought about by a variety of therapies (Melzack, 1987; Harden et al., 1991; Stelian et al., 1992). Although sensory and affective sub-scales are frequently correlated, research has provided support for their demarcation as distinct factors (Wright et al., 2001).

Anxiety Sensitivity Index (ASI; Reiss et al., 1986): The ASI is comprised of 16 items designed to assess the fear of anxiety-related sensations (e.g. 'when I notice that my heart is beating rapidly, I worry that I might have a heart attack'). Respondents indicate the degree to which each item applies to them on a 5-point scale ranging from 'very little' (0) to 'very much' (4). Items on the ASI can be summated to give an overall anxiety sensitivity score or three separate subscales, often labelled 'physical concerns', 'psychological concerns' and 'social concerns' (e.g., McWilliams et al., 2000). Reliability data for the ASI is strong, with the scale displaying strong internal consistency (Ayvasik, 2000) and test-retest reliability (Fullana et al., 2003; Lambert et al., 2004). In addition, several studies have offered support for external validity of the ASI (Maller and Reiss, 1987; Sandin et al., 1996).

Depression Anxiety Stress Scale- Short Form (DASS21; Lovibond and Lovibond, 1995b): The DASS21 is an abbreviated 21-item version of the full length DASS (Lovibond and Lovibond, 1995a). Respondents are asked to indicate on a 4-point scale, the degree to which each item applied to them over the previous week (e.g. 'I felt downhearted and blue'). Three separate subscale scores measuring the core symptoms of depression, anxiety and stress were calculated. A number of studies have supported this three factor structure (Lovibond and 
Lovibond, 1995a; Crawford and Henry, 2003), with the DASS also displaying excellent reliability and validity in both clinical and community groups (Antony et al., 1998; Clara et al., 2001).

Coping strategy selection: At the conclusion of each pain task, participants were asked to indicate whether they had used a 'focusing', 'distraction' or 'other' coping strategy. This question was included as an exploratory item to investigate any sex or anxiety sensitivity differences in the type of coping strategy employed.

\section{Blood pressure measurement}

Resting blood pressure prior to pain testing was recorded with an Omron ${ }^{\mathrm{TM}}$ automatic blood pressure monitor with a self-inflating upper-arm cuff. Participants were seated and asked to relax for a period of around five minutes, in accordance with recommended guidelines (Shapiro et al., 1996), while the experimenter performed various administrative tasks. The cuff was attached to the participant's non-dominant arm and three consecutive systolic blood pressure readings were taken, with an interval of approximately 2 minutes between each reading. An average blood pressure rating was calculated from these three readings.

\section{Procedure}

Following written consent, participants were asked to abstain from analgesics ( $48 \mathrm{hrs}$ before), alcohol (12hrs), caffeine (2hrs) and nicotine (1hr) use prior to participation. The experimental sessions took place in a temperature-controlled room maintained at $22^{\circ} \mathrm{C}$. Three blood pressure readings were taken and demographic data recorded. Pain testing then commenced with participants exposed to a single pain stimulus. Pain experience was assessed by recording threshold and tolerance times and by asking participants to complete the SF-MPQ immediately 
following noxious stimulation. The remaining questionnaire battery was then administered, and pain testing and assessment repeated on the other hand using the alternative pain stimulus.

Finally, participants reported which coping strategy they had used during exposure to the pain stimuli. The duration of the whole experiment was approximately 35 minutes, by which time the effect of the pain manipulations had dissipated. The experimental procedures were approved by Goldsmiths College Ethical Committee and conformed to ethical guidelines for pain research with humans as recommended by the International Association for the Study of Pain (1995).

\section{Results}

\section{Data screening}

Cold pain tolerance times exhibited a bimodal distribution ${ }^{1}$. The second peak of the distribution represented 18 pain tolerance times of 120 seconds (the upper time limit), nearly three standard deviations above the mean. These outliers were trimmed to the next highest value (winsorising) as recommended by Tabachnick and Fidell (2001). Altering the value of the outliers in this way to improve analysis seems reasonable given that the value of 120 seconds is merely a product of a somewhat arbitrary cut-off time. This method of dealing with extreme case values also preserves the increased pain tolerance of the outlier sample, whilst ameliorating its disproportionate influence on the data. As bimodal pain tolerance data is commonly observed in studies of sex differences in pain tolerance, analysis using the winsorised distribution was compared to analysis using bootstrapped samples of the original bimodal distribution. Specifically, the $p$-value obtained from (1) a standard t-test (IV=sex) on the winsorised

\footnotetext{
${ }^{1}$ Bimodal distributions of tolerance times are not uncommon in pain research. This is usually attributable to a small subset of participants finding the stimulus insufficiently painful to produce tolerance, which allows them to reach task cut-off points that are often well above mean tolerance times (e.g., Wolff, 1984).
} 
tolerance data was compared against the p-value obtained from (2) a t-test on the original bimodal data, but with $p$ derived not from a standard t-distribution, but from a bootstrapped distribution of 10,000 resampled t-statistics of the bimodal data (e.g., Howell, 2002). Probability values were virtually identical across the two methods, supporting the validity of the winsorising method for the current data. The cold pain threshold data exhibited a similar, if far less pronounced, bimodal pattern and were trimmed in the same way.

After winsorising cold threshold and tolerance data, histograms revealed no univariate outliers. Mahalanobis distance based on three predictors was below the critical value of $\chi^{2}(3)=16.27$, $\mathrm{p}<.001$ for every participant suggesting no multivariate outliers. A visual inspection of the histograms of all continuous variables showed that only the affective cold and heat pain measures demonstrated unacceptable departure from normality. As affective pain was positively skewed, a logarithmic transformation was applied to both scores (Tabachnick and Fidell, 2001). A check on the transformed variable showed an improved and acceptable approximation of normality. Scatterplots revealed no obvious non-linear relationships. Finally, residuals plots indicated that homoscedasticity assumptions were comfortably met for the regression analysis, and DeShon and Alexander's (1996) test indicated that residual variances were equal across male and female groups, satisfying assumptions for the simple slope analysis.

\section{$\underline{\text { Physiological controls }}$}

In order to determine whether female pain sensitivity was affected by hormonal factors, a series of $2 \times 2$ ANOVAs with IVs menstrual phase (luteal/follicular) and pill type (no pill/pill) was conducted on all five cold and all five heat pain measures for the female sub-sample. Analysis revealed no significant differences on any pain measures, even with a liberal, 
uncorrected significance level of $\alpha=.05$. As these factors appeared to have no demonstrable impact on pain sensitivity, they were not considered in the main analyses.

Sex differences in resting blood pressure and mood were also investigated, in order to determine whether these factors could potentially underlie sex differences in pain sensitivity. An independent t-test on systolic blood pressure with sex as the IV revealed significant differences in blood pressure $\left(\mathrm{t}_{121}=3.2, \mathrm{p}<.005\right)$, with males $(\mathrm{M}=120 \mathrm{mmHg})$ exhibiting higher resting blood pressure than females $(\mathrm{M}=111 \mathrm{mmHg})$. T-tests revealed no sex differences on the DASS21 subscales. Correlations of anxiety sensitivity with both resting blood pressure and the DASS21 were also conducted and revealed significant associations of anxiety sensitivity with all three DASS21 scales ( $\mathrm{r}=.39$ to $.50, \mathrm{p}<.01)$, although no association with blood pressure was observed. The potential role of both blood pressure and mood on pain rating will be considered in the main analysis below.

\section{$\underline{\text { Regression analysis on the five pain measures }}$}

A regression approach was used to investigate the relationship of anxiety sensitivity and sex with pain. Sex and anxiety sensitivity were centred (i.e. deviation scores computed by subtracting the mean of each variable from each individual score), with the interaction term the cross product of these centred variables. Centring was performed in order to reduce multicollinearity between the interaction and its constituent predictors, and to aid interpretation of the simple (main-effect) predictors (Aiken and West, 1991).

Separate standard regressions were performed on each of the five pain DVs with anxiety sensitivity, sex, and the anxiety sensitivity by sex interaction term entered as predictors. Separate regressions for each dependent pain measure were performed as, although naturally 
correlated, these measures nevertheless represent distinct pain dimensions (Price et al., 2001). To preserve the type I error rate, an $\alpha=.015$ was applied using the Dubey/Armitage-Parmar procedure (Sankoh et al., 1997). This procedure is based on Sidak's formula, but with an adjustment for the average correlation between dependent variables ${ }^{2}$, and was carried out using Uitenbroek's (1997) online SISA package. Results of the regression analyses are presented separately for cold and heat pain inductions.

\section{Cold pressor pain}

Table 1 lists the mean threshold times for males and females, along with all other pain measures for both cold and heat inductions. The overall regression model of pain threshold with sex, anxiety sensitivity and the two-way interaction approached significance at the adjusted alpha level $\left(\mathrm{R}^{2}=7.6 \%, \mathrm{~F}_{3,121}=3.32, \mathrm{p}=.022\right)$. Examination of partial regression coefficients revealed sex to be a significant predictor of cold pain threshold $\left(B=5.1, t_{121}=2.62\right.$, $\mathrm{p}<.01)$. The overall regression model for pain tolerance was significant $\left(\mathrm{R}^{2}=13.9 \%\right.$, $\left.\mathrm{F}_{3,121}=6.50, \mathrm{p}<.01\right)$. Sex was again a significant predictor of cold pain tolerance $(\mathrm{B}=15.67$, $\left.\mathrm{t}_{121}=3.69, \mathrm{p}<.001\right)$, with the mean tolerance time greater for males $(\mathrm{M}=53.7$ secs $)$ compared to females ( $M=38.6$ secs). Neither anxiety sensitivity nor the interaction term showed any significant associations with either pain threshold or tolerance.

Enter Table 1 about here

With respect to the three subjective pain measures, the overall regression model was significant for sensory pain $\left(\mathrm{R}^{2}=18.6 \%, \mathrm{~F}_{3,121}=9.19, \mathrm{p}<.01\right)$ and approached significance for affective pain $\left(\mathrm{R}^{2}=7.5 \%, \mathrm{~F}_{3,121}=3.28, \mathrm{p}=.023\right)$. For the affective pain measure, partial regression coefficients

\footnotetext{
${ }^{2}$ The average inter-item correlation between dependent variables was $r=.26$ for both cold and heat inductions.
} 
revealed anxiety sensitivity to be the only significant predictor of affective (log ) pain $(B=.02$, $\mathrm{t}_{121}=2.31$, one-tailed $\left.\mathrm{p}<.015\right)$, with higher anxiety sensitivity scores associated with higher affective pain scores. For the sensory pain measure, anxiety sensitivity was revealed to be a significant predictor $\left(\mathrm{B}=.21, \mathrm{t}_{121}=4.43, \mathrm{p}<.01\right)$. Furthermore, the ASI by sex interaction term also reached significance $\left(\mathrm{B}=.26, \mathrm{t}_{121}=2.88, \mathrm{p}<.01\right)$. In order to investigate the nature of this interaction, the relationship of anxiety sensitivity with predicted sensory pain scores was plotted separately for males and females (see Figure 1). Predicted sensory pain scores were calculated by solving the regression equation at both levels of sex for low (-1 SD), medium (0) and high (+1 SD) anxiety sensitivity scores. Figure 1 suggests that sex could act as a moderator of anxiety sensitivity, with increasing levels of anxiety sensitivity appearing to predict greater sensory pain in females but not in males. Simple slope analysis conducted using the formula reported by Cohen et al (2003, p.273), revealed that the relationship between anxiety sensitivity and sensory pain was indeed significant for females $\left(\mathrm{B}=.28, \mathrm{t}_{121}=4.87, \mathrm{p}<.01\right)$, but not for males $\left(B=.02, t_{121}=0.23, p=n . s.\right)$. No other effects reached significance on any the five DVs.

Enter Figure 1 about here

As the above effects could be associated with heightened pain purely because of an association with other negative mood state variables and/or resting blood pressure, the effect of controlling for these variables was assessed by entering the anxiety, depression and stress subscales from the DASS21 and blood pressure alongside the original predictors and re-running the regression. The same pattern of significances was observed, suggesting the relationship of anxiety sensitivity and sex with pain cannot be simply attributed to negative mood states or resting blood pressure. 


\section{Thermal heat pain}

Identical analyses to those reported above were conducted for the thermal heat induction. For the pain behaviour measures, the overall regression model with sex, anxiety sensitivity and the interaction term as predictors demonstrated significance for both heat pain threshold $\left(\mathrm{R}^{2}=11.3 \%, \mathrm{~F}_{3,121}=5.12, \mathrm{p}<.01\right)$ and tolerance $\left(\mathrm{R}^{2}=13.7 \%, \mathrm{~F}_{3,121}=6.41, \mathrm{p}<.01\right)$. Sex was a significant predictor of heat pain threshold $\left(\mathrm{B}=1.51, \mathrm{t}_{121}=3.82, \mathrm{p}<.001\right)$, with mean threshold time higher for males $(M=5.45$ secs $)$ than females $(M=3.96$ secs $)$. Sex was also a significant predictor of heat pain tolerance $\left(\mathrm{B}=1.65, \mathrm{t}_{121}=4.08, \mathrm{p}<.001\right)$, with tolerance time greater for males $(M=7.55$ secs $)$ compared to females $(M=5.90 \mathrm{secs})$. However, neither anxiety sensitivity nor the interaction term was significantly independently associated with heat pain threshold or tolerance.

For the subjective pain scores, the overall regression models were significant for all three measures $\left(\mathrm{p}<.01, \mathrm{R}^{2}=14.5-19.3 \%\right)$. Anxiety sensitivity was a significant independent predictor of affective $(\log )$ pain $\left(B=.028, \mathrm{t}_{121}=4.45, \mathrm{p}<.001\right)$, sensory pain $\left(\mathrm{B}=.183, \mathrm{t}_{121}=4.17, \mathrm{p}<.001\right)$ and PPI $\left(\mathrm{B}=.029, \mathrm{t}_{121}=3.99, \mathrm{p}<.001\right)$. The anxiety sensitivity by sex interaction term was also a significant predictor of sensory pain $\left(B=.324, \mathrm{t}_{121}=3.67, \mathrm{p}<.001\right)$ and PPI $\left(\mathrm{B}=.045, \mathrm{t}_{121}=3.15\right.$, $\mathrm{p}<.01)$. As before, inclusion of DASS and blood pressure scores did not alter the general pattern of effects found. Figures 2 and 3 present the two interactions, which suggest a possible sex-specific effect of anxiety sensitivity.

Enter Figures $2 \& 3$ about here 
Simple slope analysis revealed that, in females, anxiety sensitivity was significantly associated with sensory pain $\left(\mathrm{B}=.28, \mathrm{t}_{121}=5.05, \mathrm{p}<.001\right)$ and PPI $\left(\mathrm{B}=.04, \mathrm{t}_{121}=4.66, \mathrm{p}<.001\right)$; no significant associations were observed for males. Figure 3 also shows a mean PPI score of 2 for low anxiety-sensitive females and 2.8 for high anxiety-sensitive females. In terms of the PPI's verbal labels, these scores approximate the 'discomforting' (2) and 'distressing' (3) descriptors, suggesting the link between anxiety sensitivity and heat pain intensity in females may be substantive and meaningful. Furthermore, the partial regression coefficient ${ }^{3}$ of anxiety sensitivity and PPI in females of $B=.28$ when expressed in standardised terms was $\beta=.50$ (or $\beta^{2}=.25$ ). This suggests that a substantive $25 \%$ of the sample variance in heat PPI scores in females could be accounted for by anxiety sensitivity.

\section{Thermal heat vs. cold pain}

Results from the previous regression analysis revealed that anxiety sensitivity was associated with higher PPI ratings in females during heat but not during cold stimulation. In addition, the associations of anxiety sensitivity with sensory and affective pain generally appeared stronger for heat relative to cold. These results could indicate that anxiety sensitivity leads to a stronger subjective pain response during heat compared to cold stimulation. In order to examine this possibility, Table 2 presents the zero-order correlations between anxiety sensitivity and the subjective pain ratings for heat and cold conditions (pain threshold and tolerance were not included as they showed no association with anxiety sensitivity). In order to assess whether the

\footnotetext{
${ }^{3}$ The correct standardised partial regression coefficient $(\beta)$ was calculated, standardising sex and ASI, and entering these as predictors along with the resultant cross-product interaction term. The standard computer regression output gives a partial regression coefficient based on an interaction term formed from a cross-product of the raw scores and then standardising this cross-product term; this can result in an inaccurate beta value (Friedrich, 1982; Cohen, 2003).
} 
correlations between anxiety sensitivity and heat pain were significantly larger than the correlations between anxiety sensitivity and cold pain, Steiger's z1 test (Steiger, 1980) was performed for each of the three pain measures. This revealed that the correlations of anxiety sensitivity with both PPI $(z 1=3.05, p<.01)$ and affective pain $(z 1=2.12, p=.014)$ were significantly larger for the heat pain induction. This suggests that anxiety sensitivity may exhibit a stronger relationship with subjective pain during heat compared to cold stimulation.

Enter Table 2 about here

\section{Coping strategy and pain reports}

Given the apparent sex-specific effect of anxiety sensitivity on pain, the final analysis examined whether sex was associated with the use of a particular coping strategy. Chi-square analysis indicated a close degree of association between participants' choice of coping strategies across both cold and heat inductions $\left(\chi^{2}(2)=32.04, \mathrm{df}=2, \mathrm{~N}=125, \mathrm{p}<.0001\right)$, and so only one coping strategy variable (cold induction) was included in subsequent analysis. A chisquare analysis with sex (male/female) and coping strategy (distraction/focus/other) as variables showed a significant difference in frequency ratings $\left(\chi^{2}=8.17, \mathrm{df}=2, \mathrm{~N}=125, \mathrm{p}<.05\right)$. Three post-hoc $2 \times 2$ chi-square tests were performed at a Bonferroni adjusted alpha level of $.017(.05 / 3)$, each test omitting a different category of coping. This revealed that males were more likely to report having used a distraction strategy and females a focusing strategy $\left(\chi^{2}=6.81, \mathrm{~N}=53, \mathrm{p}<.01\right)$. No other results were significant. 


\section{Discussion}

The principal aim of the current study was to determine whether: (1) anxiety sensitivity, sex, and the interaction between the two, were linked to increased pain, and (2) this link was influenced by type of pain stimulation. As expected, sex differences in pain were found, with females reporting lower pain threshold and tolerance in response to both noxious cold and heat stimuli. This is consistent with previous studies of this type (al'Absi et al., 2004; Girdler et al., 2005; Jackson et al., 2005). Furthermore, the results showed that even after controlling for resting blood pressure, sex differences in pain tolerance remained. This suggests that even if blood pressure does contribute to sex differences in pain tolerance (e.g., Rollman et al., 2000), it is insufficient to provide a complete explanation of such differences.

Analysis also revealed anxiety sensitivity to be positively associated with affective pain for both cold and heat stimuli. Although such an association has been previously identified in studies using the cold pressor method (Keogh and Mansoor, 2001; Keogh and Cochrane, 2002), the presence of this association for both types of pain induction suggests that the relationship extends beyond cold pressor pain. In contrast to the current study, previous experimental studies that have found a link between anxiety sensitivity and affective pain appear to have consisted either exclusively or predominantly of female participants (Keogh and Mansoor, 2001; Keogh and Cochrane, 2002). The current study therefore extends the findings from these studies, and suggests that anxiety sensitivity may influence the affective pain response of males as well as females.

Anxiety sensitivity was also positively associated with sensory pain (cold and heat tasks) and pain intensity (heat task). However, such associations were observed only in females. This apparent sex-specific effect of anxiety sensitivity on sensory pain is consistent with previous 
studies utilising both experimental cold pressor inductions and naturally occurring pain (Keogh and Birkby, 1999; Keogh et al., 2004). Indeed, the fact that we found that, within women, anxiety sensitivity was positively related to sensory pain in both pain inductions also suggests that this relationship is generalisable across different types of thermal pain. However, the link between anxiety sensitivity and pain intensity appeared to be dependent upon the type of noxious stimuli administered. While anxiety sensitivity demonstrated no association with pain intensity ratings in females during cold pain stimulation, a significant association was found within females during heat pain stimulation. Furthermore, analysis of the meanings assigned to the average pain ratings revealed that, while low anxiety sensitive females reported the heat pain to be 'discomforting', high anxiety sensitive females were more likely to report the pain as 'distressing'. Interestingly, the analysis of anxiety sensitivity and affective pain scores also revealed a significantly larger association for the heat relative to the cold induction. These findings, therefore, extend previous research that has linked anxiety sensitivity to pain by suggesting that the type of noxious source could have a significant impact on the strength of the link.

Although the data do not allow for determinations of why noxious heat might produce stronger responses in high anxiety-sensitive females than noxious cold, three possible explanations are considered. Firstly, the apparent effect of noxious type may simply have reflected differences in overall perceived stimulus intensity between cold and heat inductions. However, t-tests comparing cold vs. heat pain ratings for all three subjective pain measures revealed no significant effects, suggesting no differences in perceived noxious intensity. Secondly, it may be that heat stimulation elicits greater anxiety over possible physical harm (e.g., burns, inflammation, scalding, etc.) than cold stimulation. As those high in anxiety sensitivity typically exhibit a fear of anxiety symptoms, it seems plausible they would react more 
negatively to a stimulus that produces greater anxiety and/or which is perceived to possess greater threat-value. However, although post-experimental comment appeared to support a greater relative fear of the noxious heat task, state anxiety prior to each pain induction was not quantitatively assessed. As such, this explanation remains speculative. Thirdly, the average exposure time differed substantially across inductions, with t-tests revealing contact time to be significantly greater for the cold pressor compared to the heat stimulus. It may be that the longer exposure to the cold pressor allowed the development of alternative pain coping strategies, and that these strategies may have benefited those high in anxiety sensitivity. Certainly, increasing exposure time has been previously linked to changes in the selection and effectiveness of pain-coping strategies (McCaul and Haugtvedt, 1982; Mullen and Suls, 1982). However, data from the current study are insufficient to provide an evaluation of this hypothesis, which again remains necessarily speculative.

With respect to the sex-specific effect of anxiety sensitivity, it is unclear why anxiety sensitivity appears to be associated with increased pain primarily in females. One possible source of influence is the fact that males and females have been reported to differ in their choice of pain coping strategies (Goyen and Anshel, 1998; Affleck et al., 1999; France et al., 2004). It may be that if females are more inclined to focus on anxiety-relevant sensory cues than males, this could amplify the effects of anxiety sensitivity. In fact, chi-square analysis did indicate sex differences in coping strategies, with females more likely than males to have reported the use of a focusing rather than a distraction strategy during the pain tasks. Further studies investigating whether direct manipulations of coping strategy affect the sex-specific link between anxiety sensitivity and pain are currently in progress. 
With regard to potential implications, the pattern of anxiety sensitivity effects was generally applicable across both heat and cold stimuli despite the fact that stimulation of these modalities tends to elicit diverse responses to pain ${ }^{4}$. This suggests a possible wider generalisability of anxiety sensitivity effects across other types of pain. Furthermore, the fact that stronger anxiety sensitivity effects were observed for the heat stimulus suggests that the vulnerability of high anxiety-sensitive individuals to increased pain may be exacerbated for specific types of pain. If this is the case, this suggests that therapy designed to address anxiety-sensitive cognitions could be more beneficial for some types of pain than for others. Whether such a possibility translates beyond the experimental testing situation, however, is uncertain, with the clinical applicability of the findings a notable limitation of the current work. The pain inductions used in the current study were likely to be perceived as more controllable, less anxiety producing and of lower intensity than pain that occurs in a clinical setting (Melzack and Wall, 1996). All of these factors could have a substantial impact on the generalisability of the findings outside of the laboratory. Although recent studies have identified a link between anxiety sensitivity and pain in clinical settings (Keogh et al., 2004; Lang et al., 2005), there is little research to date on whether the strength of this link is influenced by the type of clinical pain. An additional limitation is that the pain inductions differed not only in their modality, but also on a number of other dimensions. As such, it is hard to isolate the critical features of the heat stimulus responsible for the apparent heightening of anxiety sensitivity effects. Future research may, therefore, wish to extend the current study by attempting to manipulate various aspects of the stimuli (e.g. contact time) in order to identify these critical features. Studies that compare how the link between anxiety sensitivity and pain is affected by variations in clinical-based pain or surgical procedures may also provide an insightful line of further enquiry.

\footnotetext{
${ }^{4}$ Paired t-tests comparing mean scores on all 13 SF-MPQ pain descriptors across cold and heat inductions, revealed significant differences $(\mathrm{P}<.001)$ on all but 4 descriptors.
} 
In summary, the current study replicates previous research by demonstrating anxiety sensitivity to be positively associated with subjective pain ratings, with this association particularly pronounced in females. However, this study also extends previous research by suggesting the association between anxiety sensitivity and pain may be influenced by the type of pain stimulation, with noxious heat appearing to produce a stronger association than noxious cold. If this is the case, this implies that the treatment of anxiety-sensitive cognitions could be especially useful when directed towards specific types of pain. Additional research is required to identify the key features of noxious stimuli that influence the relationship between anxiety sensitivity and pain. 
Anxiety sensitivity and pain: generalisability across noxious stimuli

\section{Acknowledgements}

This research was supported by an ESRC studentship grant to the first author (award no. R42200134488). 


\section{References}

Affleck G, Tennen H, Keefe FJ, Lefebvre JC, Kashikar Zuck S, Wright K, Starr K, Caldwell DS. Everyday life with osteoarthritis or rheumatoid arthritis: Independent effects of disease and gender on daily pain, mood and coping. Pain 1999;83:601-609.

Aiken LS, West SG. Multiple regression: Testing and interpreting interactions. California, USA: Sage, 1991.

al'Absi M, Wittmers LE, Ellestad D, Nordehn G, Kim SW, Kirschbaum C, Grant JE. Sex differences in pain and hypothalamic-pituitary-adrenocortical responses to opioid blockade. Psychosom Med 2004;66:198-206.

Antony MM, Bieling PJ, Cox BJ, Enns MW, Swinson RP. Psychometric properties of the 42item and 21-item versions of the Depression Anxiety Stress Scales in clinical groups and a community sample. Psychol Assess 1998;10:176-181.

Asmundson GJ, Norton GR. Anxiety sensitivity in patients with physically unexplained chronic back pain: a preliminary report. Behav Res Ther 1995;33:771-7.

Ayvasik HB. Anxiety Sensitivity Index: Validity and reliability in Turkish sample. Turk Psikoloji Dergisi 2000;15:43-62.

Clara IP, Cox BJ, Enns MW. Confirmatory factor analysis of the Depression-Anxiety-Stress Scales in depressed and anxious patients. Journal of Psychopathology and Behavioral Assessment 2001;23:61-67.

Cohen J, Cohen P, West SG, Aiken LS. Applied multiple regression/correlation analysis for the behavioral sciences. Mahwah, NJ, US: Lawrence Erlbaum Associates, 2003. 
Crawford JR, Henry JD. The Depression Anxiety Stress Scales (DASS): Normative data and latent structure in a large non-clinical sample. Br J Clin Psychol 2003;42:111-131.

Davidson PO, McDougall CE. The generality of pain tolerance. J Psychosom Res 1969;13:839.

Dudgeon D, Raubertas RF, Rosenthal SN. The short-form McGill Pain Questionnaire in chronic cancer pain. J Pain Symptom Manage 1993;8:191-5.

Edens JL, Gil KM. Experimental induction of pain: Utility in the study of clinical pain. Behavior Therapy 1995;26:197-216.

Fillingim RB, Maixner W. The influence of resting blood pressure and gender on pain responses. Psychosom Med 1996;58:326-332.

France CR, Keefe FJ, Emery CF, Affleck G, France JL, Waters S, Caldwell DS, Stainbrook D, Hackshaw KV, Edwards C. Laboratory pain perception and clinical pain in post-menopausal women and age-matched men with osteoarthritis: Relationship to pain coping and hormonal status. Pain 2004;112:274-281.

Fullana MA, Servera M, Weems CF, Tortella Feliu M, Caseras X. Psychometric properties of the Childhood Anxiety Sensitivity Index in a sample of Catalan school children. Anxiety, Stress and Coping: An International Journal 2003;16:99-107.

Girdler SS, Maixner W, Naftel HA, Stewart PW, Moretz RL, Light KC. Cigarette smoking, stress-induced analgesia and pain perception in men and women. Pain 2005;114:372-385. 
Goyen MJ, Anshel MH. Sources of acute competitive stress and use of coping strategies as a function of age and gender. Journal of Applied Developmental Psychology 1998;19:469-486.

Harden RN, Carter TD, Gilman CS, Gross AJ, Peters JR. Ketorolac in acute headache management. Headache 1991;31:463-4.

Howell DC. Statistical Methods for Psychology. Pacific Grove, CA: Duxbury, 2002.

IASP. Ethics, human experiments (statement). Retrieved 26 Feb, 2003, from the World Wide Web: http://www.iasp-pain.org/ethics-h.html. 1995.

Jackson T, Iezzi T, Chen H, Ebnet S, Eglitis K. Gender, interpersonal transactions, and the perception of pain: An experimental analysis. J Pain 2005;6:228-236.

Janal MN, Glusman M, Kuhl JP, Clark WC. On the absence of correlation between responses to noxious heat, cold, electrical and ischemic stimulation. Pain 1994;58:403-11.

Jones A, Zachariae R, Arendt Nielsen L. Dispositional anxiety and the experience of pain: gender-specific effects. Eur J Pain 2003;7:387-395.

Keogh E, Birkby J. The effect of anxiety sensitivity and gender on the experience of pain. Cognition and Emotion 1999;13:813-829.

Keogh E, Cochrane M. Anxiety sensitivity, cognitive biases, and the experience of pain. J Pain $2002 ; 3: 320-329$.

Keogh E, Hamid R, Hamid S, Ellery D. Investigating the effect of anxiety sensitivity, gender and negative interpretative bias on the perception of chest pain. Pain 2004;111:209-217. 
Keogh E, Mansoor L. Investigating the effects of anxiety sensitivity and coping on the perception of cold pressor pain in healthy women. Eur J Pain 2001;5:11-22.

Lambert SF, Cooley MR, Campbell KDM, Benoit MZ, Stansbury R. Assessing anxiety sensitivity in inner-city African American children: Psychometric properties of the Childhood Anxiety Sensitivity Index. J Clin Child Adolesc Psychol 2004;33:248-259.

Lang AJ, Sorrell JT, Rodgers CS, Lebeck MM. Anxiety sensitivity as a predictor of labor pain. Eur J Pain 2005;10:263-270.

Lovibond PF, Lovibond SH. The structure of negative emotional states: Comparison of the Depression Anxiety Stress Scales (DASS) with the Beck Depression and Anxiety Inventories. Behav Res Ther 1995a;33:335-343.

Lovibond SH, Lovibond PF. Manual for the Depression Anxiety Stress Scales. Sydney: The Psychology Foundation of Australia, UNSW, 1995 b.

Lynn B, Perl ER. A comparison of four tests for assessing the pain sensitivity of different subjects and test areas. Pain 1977;3:353-65.

Maller RG, Reiss S. A behavioral validation of the Anxiety Sensitivity Index. J Anxiety Disord $1987 ; 1: 265-272$.

McCaul KD, Haugtvedt C. Attention, distraction, and cold-pressor pain. J Pers Soc Psychol $1982 ; 43: 154-62$. 
McWilliams LA, Stewart SH, MacPherson PSR. Does the social concerns component of the Anxiety Sensitivity Index belong to the domain of anxiety sensitivity or the domain of negative evaluation sensitivity. Behav Res Ther 2000;38:985-992.

Melzack R. The short-form McGill Pain Questionnaire. Pain 1987;30:191-7.

Melzack R, Wall PD. The Challenge of Pain. London, England: Penguin Books Ltd, 1996.

Mullen B, Suls J. The effectiveness of attention and rejection as coping styles: a meta-analysis of temporal differences. J Psychosom Res 1982;26:43-9.

Plehn K, Peterson RA, Williams DA. Anxiety sensitivity: Its relationship to functional status in patients with chronic pain. J Occup Rehabil 1998;8:213-222.

Price DD, Riley JL, Wade JB. Psychophysical approaches to measurement of the dimensions and stages of pain. In: Turk DC, Melzack R, editors. Handbook of Pain Assessment. London: The Guilford Press, 2001. pp. 53-75.

Reiss S, Peterson RA, Gursky DM, McNally RJ. Anxiety sensitivity, anxiety frequency and the predictions of fearfulness. Behav Res Ther 1986;24:1-8.

Riley JL, III, Robinson ME, Wise EA, Myers CD, Fillingim RB. Sex differences in the perception of noxious experimental stimuli: A meta-analysis. Pain 1998;74:181-187.

Riley JL, III, Robinson ME, Wise EA, Price DD. A meta-analytic review of pain perception across the menstrual cycle. Pain 1999;81:225-235. 
Rollman GB, Lautenbacher S, Jones KS. Sex and gender differences in responses to experimentally induced pain in humans. In: Fillingim RB, editors. Sex, gender, and pain. Progress in pain research and management, vol 17. Seattle, WA: IASP Press, 2000. pp. 165190.

Sandin B, Chorot P, McNally RJ. Validation of the Spanish version of the Anxiety Sensitivity Index in a clinical sample. Behav Res Ther 1996;34:283-290.

Sankoh AJ, Huque MF, Dubey SD. Some comments on frequently used multiple endpoint adjustment methods in clinical trials. Stat Med 1997;16:2529-42.

Shapiro D, Jamner LD, Lane JD, Light KC, Myrtek M, Sawada Y, Steptoe A. Blood pressure publication guidelines. Psychophysiology 1996;33:1-12.

Steiger JH. Tests for comparing elements of a correlation matrix. Psychol Bull 1980;82:245251.

Stelian J, Gil I, Habot B, Rosenthal M, Abramovici I, Kutok N, Khahil A. Improvement of pain and disability in elderly patients with degenerative osteoarthritis of the knee treated with narrow-band light therapy. J Am Geriatr Soc 1992;40:23-6.

Tabachnick BG, Fidell LS. Using Multivariate Statistics. New York: Harper Collins, 2001.

Taylor S. Anxiety sensitivity: Theory, research, and treatment of the fear of anxiety. Mahwah, US: Lawrence Erlbaum Associates. 1999.

Uitenbroek DG. SISA-Bonferroni. Retrieved 13th April, 2006, from the World Wide Web: http://home.clara.net/sisa/bonfer.htm. 1997. 
Wolff B. Methods of testing pain mechanisms in normal man. In: Wall PD, Melzack R, editors. Textbook of pain. Edinburgh: Churchill Livingstone, 1984. pp. 186-194.

Wright KD, Asmundson GJ, McCreary DR. Factorial validity of the Short-Form McGill Pain Questionnaire (SF-MPQ). Eur J Pain 2001;5:279-84. 
Table 1. Sex differences in all pain variables for each type of pain induction (SDs in parentheses).

\begin{tabular}{llccccc}
\hline \hline Modality & Sex & Threshold & Tolerance & PPI & Sensory & Affective \\
\hline Cold & Female & $15.4(10.0)$ & $38.6(22.9)$ & $2.4(0.9)$ & $9.2(5.3)$ & $1.6(2.5)$ \\
& Male & $20.4(9.5)$ & $53.7(19.0)$ & $2.7(0.6)$ & $11.0(4.2)$ & $2.1(2.3)$ \\
Heat & Female & $4.0(1.7)$ & $5.9(1.9)$ & $2.4(0.8)$ & $8.6(5.0)$ & $1.4(1.9)$ \\
& Male & $5.5(2.6)$ & $7.6(2.4)$ & $2.4(0.7)$ & $10.1(4.2)$ & $1.5(1.7)$ \\
& & & & & \\
\hline
\end{tabular}


Table 2. Pearson correlations between anxiety sensitivity, heat and cold pain ratings for subjective pain ratings.

\begin{tabular}{lccc}
\hline \hline & PPI $^{\mathrm{a}}$ & Sensory Pain & Affective Pain $^{\mathrm{b}}$ \\
\hline \hline ASI (heat pain) & $.44^{*}$ & $.47^{*}$ & $.37^{*}$ \\
ASI (cold pain) & .09 & $.46^{*}$ & $.24^{*}$ \\
heat pain - cold pain & $.39^{*}$ & $.57^{*}$ & $.72^{*}$ \\
${ }^{\mathrm{a}} \mathrm{N}=88$. Only female PPI and sensory pain data were used, as ASI showed no \\
association on these variables in males. ${ }^{\mathrm{b}} \mathrm{N}=125 .{ }^{*} \mathrm{p}<.01$. \\
\hline \hline
\end{tabular}

Figure 1. Predicted sensory pain scores across levels of anxiety sensitivity and sex for cold pain induction. 
Figure 2. Predicted sensory scores across levels of anxiety sensitivity and sex for heat pain induction. 
Figure 3. Predicted PPI scores across levels of anxiety sensitivity and sex for heat pain induction. 